Case Report

\title{
Hirsutism Caused by Pregnancy Luteoma in a Low-Resource Setting: A Case Report and Literature Review
}

\author{
David Hamisi Mvunta $\left(\mathrm{D},{ }^{1,2}\right.$ Fatemazahra Amiji $(\mathrm{D}),{ }^{1}$ Mubina Suleiman $(\mathrm{D}),{ }^{1,3}$ \\ Francisco Baraka $\mathbb{D}^{1,4}$ Ikrah Abdallah $\mathbb{D}^{1,},{ }^{1,5}$ Mabula Kazabula, ${ }^{6}$ Peter J. T. Wangwe $\mathbb{D}^{1}{ }^{1}$ \\ and Furaha August $\mathbb{D}^{1}$ \\ ${ }^{1}$ Department of Obstetrics and Gynecology, Muhimbili University of Health and Allied Sciences, 9 United Nations Road, \\ Upanga West, P.O. Box 65017, Dar es Salaam, Tanzania \\ ${ }^{2}$ Department of Obstetrics and Gynecology, Mawenzi Regional Referral Hospital, P.O. Box 3054, Moshi, Tanzania \\ ${ }^{3}$ Department of Obstetrics and Gynecology, Mnazi Mmoja Hospital, P.O. Box 236, Zanzibar, Tanzania \\ ${ }^{4}$ Department of Obstetrics and Gynecology, Maweni Regional Referral Hospital, P.O. Box 16, Kigoma, Tanzania \\ ${ }^{5}$ Department of Obstetrics and Gynecology, Kondoa District Hospital, P.O. Box 40, Dodoma, Tanzania \\ ${ }^{6}$ Department of Obstetrics and Gynecology, Lugalo Military Hospital, P.O. Box 60126, Mwenge, Dar es Salaam, Tanzania
}

Correspondence should be addressed to David Hamisi Mvunta; davims85@gmail.com

Received 25 November 2020; Revised 4 March 2021; Accepted 17 March 2021; Published 25 March 2021

Academic Editor: Daniel Martin

Copyright (C) 2021 David Hamisi Mvunta et al. This is an open access article distributed under the Creative Commons Attribution License, which permits unrestricted use, distribution, and reproduction in any medium, provided the original work is properly cited.

\begin{abstract}
Background. Pregnancy luteomas are rare, benign, ovarian neoplasms resulting from increased androgenic activity during pregnancy. Often, they occur asymptomatically and are only diagnosed incidentally during imaging or surgery: cesarean section or postpartum tubal ligation. Most common symptoms associated with pregnancy luteoma include acne, deepening of voice, hirsutism, and clitoromegaly. Most pregnancy luteomas regress spontaneously postpartum. Thus, the management of pregnancy luteomas depends on the clinical situation. Case. We report a case of 28 -year-old gravida 2, para 1 who presented at $39+1$ weeks of gestation with prolonged labor and delivered by emergency cesarean. Intraoperatively, a huge left ovarian mass was identified and resected, and tissue was sent for histopathology and a diagnosis of pregnancy luteoma was made after the pathological report. Conclusion. The present report emphasizes that pregnancy luteoma is a benign neoplasm and imprudent surgical intervention should be reserved. Proper imaging techniques, preferably MRI or ultrasonography that visualize the size of the ovary and reproductive hormonal profiles, would suffice for the diagnosis and management of pregnancy luteoma.
\end{abstract}

\section{Introduction}

Pregnancy luteoma is a rare nonneoplastic tumor-like lesion of the ovary that has an increased androgenic activity during pregnancy [1]. It is associated with varied symptoms, for instance, hirsutism, acne, deepening of voice, and virilization symptoms [2]. The first occurrence was reported by Sternberg and Barclay in 1966, and to date, very few cases have been documented in the literature [3]. It is almost always incidentally identified during operation, either at the time of cesarean section or during postpartum tubal ligation [3]. Many cases have been documented to resolve postpartum, but some have been associated with recurrence in the subsequent pregnancy [4]. Accurate case diagnosis is pertinent to avoid unnecessary oophorectomy, which may result in grave effects in the subsequent years. We present a woman incidentally found to have a large solid ovarian mass that presented a diagnostic dilemma intraoperatively and confirmed only after a pathological report.

\section{Case Report}

A 28-year-old pregnant woman with $\mathrm{BMI}>30$, gravida 2, para 1 , at 39 weeks and 1 day of gestation, was admitted to the antenatal ward presenting with lower abdominal pain 


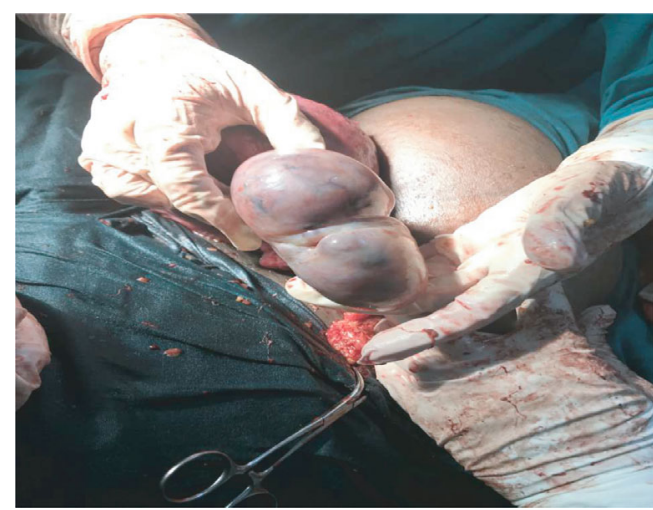

(a)

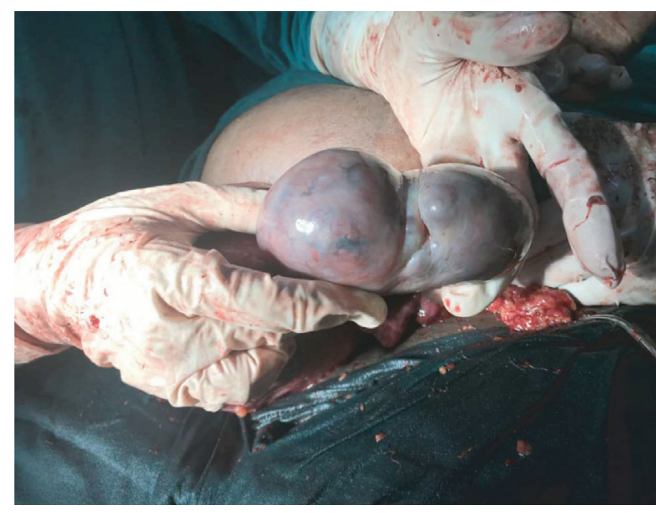

(c)

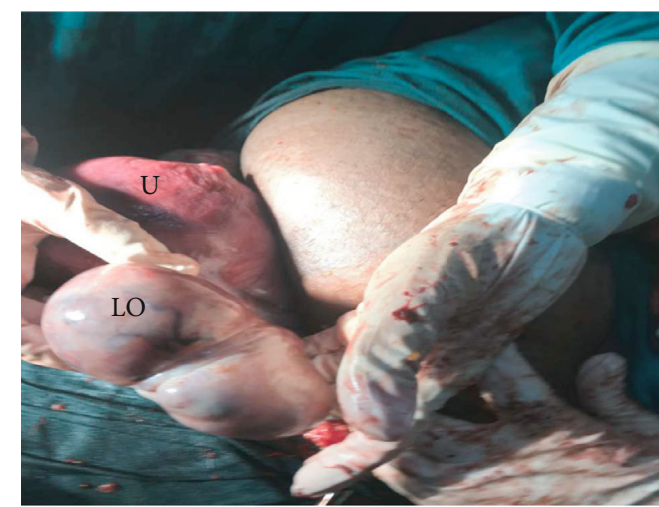

(b)

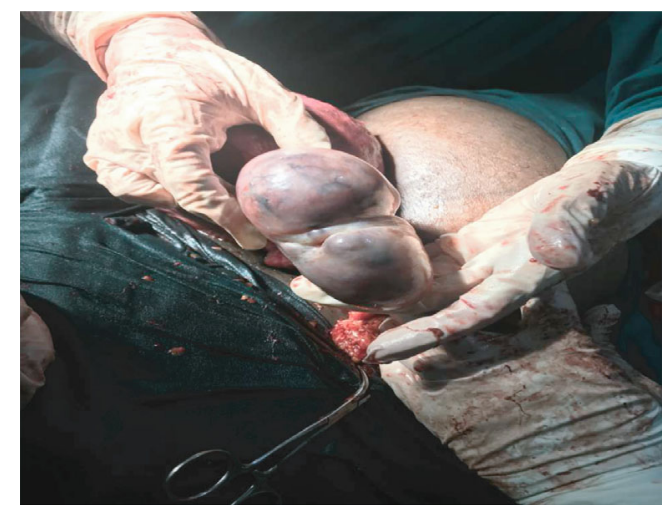

(d)

Figure 1: (a-d) Photographs showing grossly enlarged left ovary. (b) Demonstrates the relations of the enlarged left ovary (LO) to the uterus (U).

and mucoid bloody stained discharge. Her current antenatal, medical, and gynecological history was unremarkable; menarche was during her $10^{\text {th }}$ year; and her menses were painless and of regular length, amount, and flow. During the index pregnancy, nothing remarkable was noted during her antenatal visits. She did two obstetric ultrasounds at 31 and 37 weeks, and both showed a viable fetus with no adnexal masses.

Past obstetric history is remarkable for preeclampsia from the $24^{\text {th }}$ week of pregnancy and vaginal delivery of a premature male stillbirth of $1.5 \mathrm{~kg}$ about 2 years ago. She acknowledged having facial hirsutism that started from the $20^{\text {th }}$ week of pregnancy and persisted postpartum but was not associated with deepening of the voice or other virilization symptoms.

On general examination, she had normal findings, with normal female hair distribution except for facial hair at the chin, and no other signs of virilization were noted. She reported that acne was present earlier in pregnancy but subsided as the pregnancy progressed.

2.1. Labor and Delivery. Her antenatal work-up revealed she was in the latent phase of labor and was kept for observation. Following an active onset of labor, she was transferred to the labor ward, but her labor progressed poorly and was prepared for emergency cesarean. Intraoperatively, a healthy female infant (with no virilization features) weighing $3.5 \mathrm{~kg}$ with Apgar scores of 9 and 10 at the first and fifth minutes, respectively, was extracted, and the uterus was then repaired accordingly. During the repair, a huge mass on the left adnexa measuring about $9 \mathrm{~cm} \times 7 \mathrm{~cm} \times 5.5 \mathrm{~cm}$ involving the left ovary was noted (Figures $1(\mathrm{a})-1(\mathrm{~d})$ ), and unilateral oophorectomy was performed. The whole ovarian tissue (Figures 2(a) and 2(b)) was then sent for permanent section histopathological assessment suspecting ovarian malignancy. The uterus and right ovary both appeared macroscopically normal.

The gross pathological examination revealed a large ovarian tissue with two grossly visible yellowish tumor-like marked granular cysts measuring $1.5 \mathrm{~cm}$ by $1 \mathrm{~cm}$ and $1 \mathrm{~cm}$ by $1 \mathrm{~cm}$. On histology, there were granular luteinized cells in both cell masses but were otherwise normal ovarian tissues with hemorrhages and two follicular cysts with 2-3 mitoses/10 HPF (Figures 3(a) and 3(b)). Finally, it was diagnosed as pregnancy luteoma, with no obvious malignancy.

2.2. Literature Review. We reviewed various pregnancy luteoma case reports published in English between 2000 and 2020. Our review included studies obtained from reference to published articles and literature search engines: PubMed or Google Scholar, using the terms "pregnancy luteoma." A total of 205 case reports were reviewed from PubMed and 2,100 from Google Scholar. We identified and summarized 24 articles reporting 25 cases of pregnancy luteoma [2, 4-25] (Table 1). 


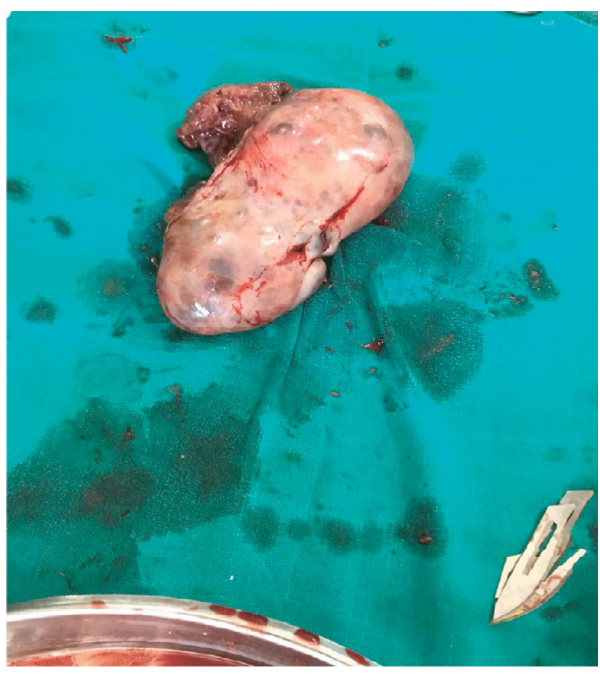

(a)

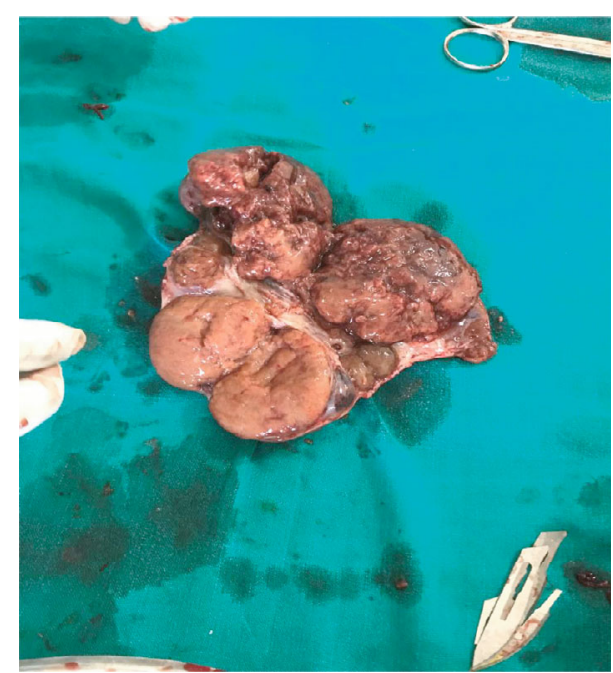

(b)

Figure 2: (a, b) A whole section of the enlarged left ovary after unilateral oophorectomy.

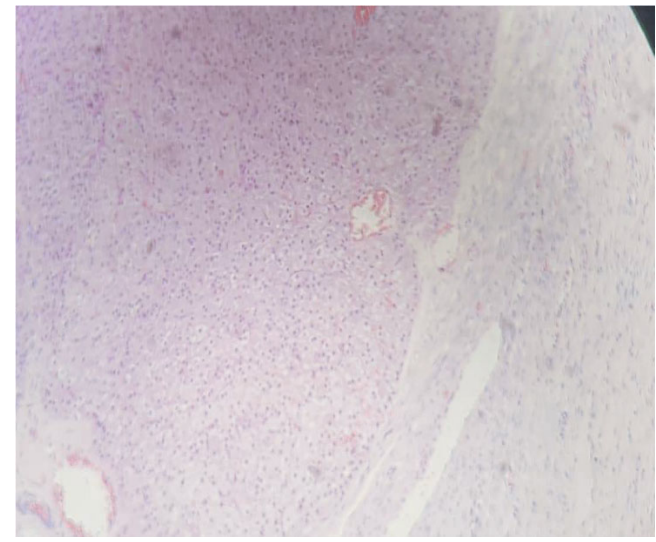

(a)

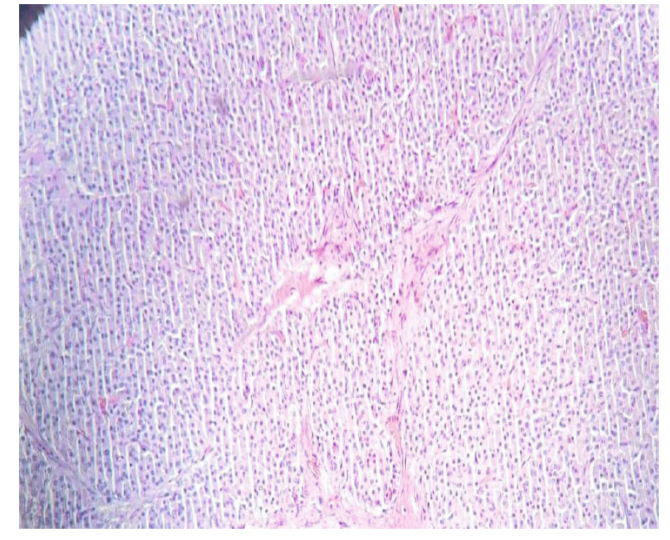

(b)

Figure 3: $(\mathrm{a}, \mathrm{b})$ Microscopic view of ovarian tissue after $\mathrm{H}$ and $\mathrm{E}$ stain.

\section{Discussion}

Pregnancy luteoma is a rare benign tumor-like enlargement of the ovary accompanying pregnancy [4]. An occurrence preponderance has been noted among the Afro-Caribbean around the ages of 30 and 40 years old and those with preexisting PCOS [11]. PCOS contributes to high $\beta$-hCG hormone levels favoring the proliferation of pregnancy luteoma $[2,26]$. These findings on age of occurrence are consistent with our case report. The present literature review adds evidence to this finding on age of occurrence (Table 1). In addition, we found the occurrence not to be specific to the AfroCaribbean but rather multiracial (Table 1). The exact age of incidence is unknown since most patients are asymptomatic and often incidentally diagnosed intraoperatively during cesarean or postoperative tubal ligation [4]. Furthermore, pregnancy luteoma masses have been reported to cause dystocia $[7,27]$, a finding that could have occurred in our case since C-section was done due to poor progress of labor.
The etiology of pregnancy luteoma, although unclear, is proposed to arise from the proliferation of theca-lutein cells following hormonal stimulation [3].

Patients with pregnancy luteoma have been reported to present with features of androgen excess, for instance, acne, deepening of the voice, facial and/or abdominal hirsutism, and clitoromegaly [4]. These symptoms have been reported to begin sometime during pregnancy and subside or stop during the postpartum period (Table 1). Unfortunately, in other patients, not all of the symptoms subside: hirsutism, deepening of the voice, and clitoromegaly seem to persist while acne and hair loss subside [4]. In the present case report, the time for follow-up was short to ascertain whether there was subsiding of the mentioned symptom of hirsutism. This is a limitation in our case report. Several authors have shown virilization symptoms in the female fetus [11], a finding that was absent in our case report. It is hypothesized that despite the maternal hyperandrogenemia produced by the pregnancy luteoma between the critical 9-14 weeks of 
TABLE 1: Literature review of pregnancy luteoma case reports: from 2000 to 2020.

\begin{tabular}{|c|c|c|c|c|c|c|}
\hline $\begin{array}{l}\text { Case } \\
\#\end{array}$ & Patient & Author (year) & $\begin{array}{l}\text { Presenting symptom } \\
\text { \& investigations }\end{array}$ & Management offered & $\begin{array}{c}\text { Outcome } \\
\text { (fetal genitalia } \\
\text { effects) }\end{array}$ & $\begin{array}{l}\text { Country } \\
\text { (race) }\end{array}$ \\
\hline 1 & $\begin{array}{c}28 \text { yr old, G2P1, } \\
\text { GA } 39 \mathrm{w}\end{array}$ & Our case report & $\begin{array}{l}\text { (1) Incidental operative } \\
\text { finding with hirsutism } \\
\text { (2) USS-done but not } \\
\text { seen, hormonal } \\
\text { assays-not done }\end{array}$ & $\begin{array}{l}\text { Cesarean (unrelated to } \\
\text { luteoma) } \\
\text { +left oophorectomy }\end{array}$ & $\begin{array}{l}\text { Female infant } \\
\text { (nil) }\end{array}$ & $\begin{array}{l}\text { Tanzania } \\
\text { (African) }\end{array}$ \\
\hline 2 & $\begin{array}{c}25 \text { yr old, G1P0, } \\
\text { GA } 28 \mathrm{w}\end{array}$ & $\begin{array}{l}\text { Rapisarda } \\
\text { et al. (2016) }\end{array}$ & $\begin{array}{l}\text { (1) Facial acne, } \\
\text { abdominal \& facial } \\
\text { hirsutism start GA } 23 \mathrm{w} \\
\text { (2) USS-right adnexal } \\
\text { mass, elevated male } \\
\text { hormones (testosterone, } \\
\text { DHEAS, SHBG, } \\
\text { androstenedione }\end{array}$ & $\begin{array}{l}\text { Conservative: till worsened } \\
\text { clinical situation and } \\
\text { cesarean @ } 34 \mathrm{w} \\
\text { GA+right oophorectomy }\end{array}$ & $\begin{array}{l}\text { Male infant } \\
\quad \text { (nil) }\end{array}$ & $\begin{array}{c}\text { Italy } \\
\text { (Spanish) }\end{array}$ \\
\hline 3 & $\begin{array}{l}28 \mathrm{yr} \text { old, G1P0, GA } \\
28 \mathrm{w}\end{array}$ & $\begin{array}{l}\text { Masarie et al. } \\
\quad(2010)\end{array}$ & $\begin{array}{l}\text { (1) Incidental finding on } \\
33 \mathrm{w} \text { USS, virilization } \\
\text { (2) Elevated testosterone }\end{array}$ & Cesarean@37w & $\begin{array}{l}\text { Female infant } \\
\text { (nil) }\end{array}$ & $\begin{array}{c}\text { USA } \\
\text { (Latina) }\end{array}$ \\
\hline 4 & G1P0 & Wang et al. (2005) & $\begin{array}{l}\text { (1) Dysuria, left flank } \\
\text { pain, fever @ } 35 \text { w } \\
\text { (2) Imaging-bilateral } \\
\text { adnexa masses } \\
\text { +hydronephrosis, } \\
\text { elevated testosterone }\end{array}$ & $\begin{array}{c}\text { Conservative: vaginal } \\
\text { delivery @ 36w }\end{array}$ & $\begin{array}{l}\text { Female infant } \\
\text { +virilization } \\
\text { (clitoromegaly) }\end{array}$ & Taiwan \\
\hline 5 & $\begin{array}{l}26 \mathrm{yr} \text { old, G1P0, GA } \\
35 \mathrm{w}\end{array}$ & Kao et al. (2005) & $\begin{array}{l}\text { (1) Deepened voice, } \\
\text { hirsutism, Elevated } \\
\text { testosterone } \\
\text { (2) USS \& MRI-bilateral } \\
\text { ovarian enlargement }\end{array}$ & $\begin{array}{l}\text { Conservative: vaginal delivery } \\
\text { @ } 36 \mathrm{w} \\
3^{\text {rd }} \text { week postpartum, } \\
\text { testosterone normalized; } 2 \\
\text { months later, ovarian masses } \\
\text { normalized, hirsutism } \\
\text { improved but fetal } \\
\text { clitoromegaly persisted }\end{array}$ & $\begin{array}{l}\text { Female infant } \\
\text { +virilization } \\
\text { (clitoromegaly) }\end{array}$ & Taiwan \\
\hline 6 & $28 \mathrm{yr}$ old, full-term & $\begin{array}{l}\text { Kumar et al. } \\
\qquad(2014)\end{array}$ & $\begin{array}{l}\text { (1) Incidental operative } \\
\text { finding } \\
\text { (2) USS-enlarged left } \\
\text { ovary, } \\
\text { hormonal studies-not } \\
\text { done }\end{array}$ & $\begin{array}{c}\text { Cesarean+right salpingo- } \\
\text { oophorectomy }\end{array}$ & Fetus (nil) & India \\
\hline 7 & $\begin{array}{c}23 \mathrm{yr} \text { old, G1P0, GA } \\
22 \mathrm{w}\end{array}$ & $\begin{array}{l}\text { Tannus et al. } \\
\text { (2009) }\end{array}$ & $\begin{array}{l}\text { (1) Incidental USS } \\
\text { finding @ } 22 \mathrm{w} \\
\text { (2) USS \& MRI-right } \\
\text { ovarian mass }\end{array}$ & $\begin{array}{l}\text { Conservative: induction of } \\
\text { labor due to postdate; } \\
\text { following failed induction } \\
\text { cesarean+right } \\
\text { oophorectomy }\end{array}$ & $\begin{array}{l}\text { Male infant } \\
\quad \text { (nil) }\end{array}$ & USA \\
\hline
\end{tabular}


TABle 1: Continued.

\begin{tabular}{|c|c|c|c|c|c|c|}
\hline $\begin{array}{l}\text { Case } \\
\#\end{array}$ & Patient & Author (year) & $\begin{array}{l}\text { Presenting symptom } \\
\text { \& investigations }\end{array}$ & Management offered & $\begin{array}{c}\text { Outcome } \\
\text { (fetal genitalia } \\
\text { effects) }\end{array}$ & $\begin{array}{l}\text { Country } \\
\text { (race) }\end{array}$ \\
\hline \multirow{3}{*}{8} & \multirow{3}{*}{$\begin{array}{c}32 \mathrm{yr} \text { old, G2P0, GA } \\
32 \mathrm{w}\end{array}$} & \multirow{3}{*}{ Dahl et al. (2008) } & $\begin{array}{l}\text { (1) Deepened voice @ } \\
32 \text { w, balding, } \\
\text { clitoromegaly, hirsutism }\end{array}$ & $\begin{array}{l}\text { Conservative to } 36 \mathrm{w} \text { and } \\
\text { cesarean (unrelated to } \\
\text { luteoma) }\end{array}$ & \multirow[t]{3}{*}{ Male infant } & \multirow[t]{3}{*}{ USA } \\
\hline & & & (2) Elevated testosterone & & & \\
\hline & & & $\begin{array}{l}\text { (3) USS \& MRI-ovaries } \\
\text { not visualized }\end{array}$ & $\begin{array}{c}\text { Ovaries were reserved, } \\
\text { developed lactational delay } \\
\text { of } 1 \mathrm{w}\end{array}$ & & \\
\hline 9 & $\begin{array}{l}21 \text { yr old, } 29 \text { yr old, } \\
\text { G1P0, GA } 34 \mathrm{w}\end{array}$ & $\begin{array}{l}\text { Nanda et al. } \\
\text { (2014) }\end{array}$ & $\begin{array}{l}\text { (1) Incidental USS } \\
\text { finding-bilateral ovarian } \\
\text { masses }\end{array}$ & $\begin{array}{c}\text { Cesarean (unrelated to } \\
\text { luteoma)+bilateral } \\
\text { oophorectomy }\end{array}$ & $\begin{array}{l}\text { Female infant } \\
\text { (nil) }\end{array}$ & Oman \\
\hline \multirow[t]{2}{*}{10} & \multirow{2}{*}{$\begin{array}{l}30 \text { yr old, } \\
\text { amenorrhoeic for } \\
2 \text { months }\end{array}$} & \multirow[t]{2}{*}{ Brar et al. (2017) } & $\begin{array}{l}\text { (1) Feature suggestive of } \\
\text { ectopic pregnancy } \\
\text { (abdominal pain, } \\
\text { vomiting) }\end{array}$ & \multirow[t]{2}{*}{$\begin{array}{l}\text { Explorative laparotomy for } \\
\text { raptured ectopic pregnancy } \\
\text { +salpingo-oophorectomy }\end{array}$} & - & India \\
\hline & & & $\begin{array}{l}\text { (2) USS-raptured tubal } \\
\text { ectopic pregnancy, solid } \\
\text { right ovarian mass }\end{array}$ & & & \\
\hline 11 & $\begin{array}{c}28 \text { yr old, G1P0, } \\
\text { GA } 42 \mathrm{w}\end{array}$ & Roth et al. (2000) & $\begin{array}{l}\text { Incidental operative } \\
\text { finding }\end{array}$ & $\begin{array}{c}\text { Cesarean after failed } \\
\text { induction }\end{array}$ & $\begin{array}{l}\text { Infant with } \\
\text { ambiguous } \\
\text { genitalia } \\
\text { +virilization } \\
\text { (clitoromegaly) }\end{array}$ & German \\
\hline \multirow{5}{*}{12} & \multirow{5}{*}{$\begin{array}{c}36 \text { yr old, G1P0, } \\
\text { conceived following } \\
\text { IVF }\end{array}$} & \multirow{5}{*}{$\begin{array}{l}\text { Spitzer et al. } \\
\quad(2007)\end{array}$} & $\begin{array}{l}\text { (1) Features of GDM, } \\
\text { gestational HTN }\end{array}$ & $\begin{array}{l}\text { Assisted vaginal delivery } \\
\text { (unrelated to luteoma) } \\
\text { @ } 36 \mathrm{w}\end{array}$ & $\begin{array}{l}\text { Female infant } \\
\text { with } \\
\text { ambiguous }\end{array}$ & USA \\
\hline & & & $\begin{array}{l}\text { (2) USS-solid lesions } \\
\text { suggesting fibroids (from } \\
6^{\text {th }} \text { to } 29^{\text {th }} \text { w GA) }\end{array}$ & $\begin{array}{c}\text { On postpartum D- } 18 \\
\text { laparotomy+omentectomy } \\
\text { +right salpingo- } \\
\text { oophorectomy done }\end{array}$ & \multirow[t]{4}{*}{ Genitalia } & \\
\hline & & & $\begin{array}{l}\text { (3) Review of maternal } \\
\text { hx: acne, deepening of } \\
\text { voice, hirsutism, } \\
\text { clitoromegaly }\end{array}$ & $\begin{array}{c}\text { (prenatal fibroids not seen, } \\
\text { probably were enlarged } \\
\text { ovaries) }\end{array}$ & & \\
\hline & & & $\begin{array}{l}\text { (4) Labs; elevated } \\
\text { testosterone }\end{array}$ & & & \\
\hline & & & $\begin{array}{l}\text { (5) USS on } 12^{\text {th }} \\
\text { postpartum day-complex } \\
\text { right ovary }\end{array}$ & $\begin{array}{c}\text { Frozen sections suggested } \\
\text { stromal tumor, but final } \\
\text { pathological report confirmed } \\
\text { luteoma }\end{array}$ & & \\
\hline \multirow[t]{3}{*}{13} & \multirow{3}{*}{$\begin{array}{l}39 \text { yr old, G2P1, hx of } \\
\text { primary subfertility } \\
\text { and underwent a } \\
\text { wedge resection of left } \\
\text { ovary for PCOD }\end{array}$} & \multirow{3}{*}{$\begin{array}{l}\text { Banerjee et al. } \\
\quad(2006)\end{array}$} & $\begin{array}{l}\text { (1) Uneventful during } \\
\text { with episodes of } \\
\text { threatened miscarriage } \\
\text { and pre-eclampsia, sickle } \\
\text { cell gene carrier }\end{array}$ & \multirow[t]{3}{*}{ Cesarean+left cystectomy } & \multirow[t]{3}{*}{ Male infant } & \multirow[t]{3}{*}{ UK (black) } \\
\hline & & & $\begin{array}{l}\text { (2) USS @ } 12^{\text {th }} \& 20^{\text {th }} w \\
\text { GA-no adnexal masses }\end{array}$ & & & \\
\hline & & & $\begin{array}{l}\text { (3) Incidental operative } \\
\text { finding }\end{array}$ & & & \\
\hline
\end{tabular}


TABle 1: Continued.

\begin{tabular}{|c|c|c|c|c|c|c|}
\hline $\begin{array}{l}\text { Case } \\
\#\end{array}$ & Patient & Author (year) & $\begin{array}{l}\text { Presenting symptom } \\
\text { \& investigations }\end{array}$ & Management offered & $\begin{array}{c}\text { Outcome } \\
\text { (fetal genitalia } \\
\text { effects) }\end{array}$ & $\begin{array}{l}\text { Country } \\
\text { (race) }\end{array}$ \\
\hline 14 & $\begin{array}{l}33 \text { yr old, G1P0, GA } \\
35 \mathrm{w}\end{array}$ & Ugaki et al. (2009) & $\begin{array}{l}\text { (1) Retrospectively study } \\
\text { of hx, hair loss, } \\
\text { hirsutism, deepening of } \\
\text { voice } \\
\text { (2) TUSS-left ovarian } \\
\text { tumor }\end{array}$ & Cesarean+left cystectomy & $\begin{array}{l}\text { Female infant } \\
\text { +virilization } \\
\text { (clitoromegaly) }\end{array}$ & Japan \\
\hline 15 & $\begin{array}{l}33 \text { yr old, G1P0, GA } \\
33 \mathrm{w}\end{array}$ & Tan et al. (2008) & $\begin{array}{l}\text { (1) Features of raptured } \\
\text { ovarian torsion: severe } \\
\text { abdominal pain and } \\
\text { decreasing Hb } \\
\text { @ GA } 33 \mathrm{w} \text { and treated } \\
\text { but recurred after } \\
\text { delivery @ } 36 \mathrm{w} \text { GA } \\
\text { (2) USS-enlarged right } \\
\text { adnexa mass probably } \\
\text { ovarian with } \\
\text { intratumoral bleeding }\end{array}$ & $\begin{array}{l}\text { Diagnostic laparotomy+right } \\
\text { salpingo-oophorectomy done }\end{array}$ & Not mentioned & $\begin{array}{l}\text { Singapore } \\
\text { (Indian) }\end{array}$ \\
\hline \multirow{3}{*}{16} & \multirow[t]{2}{*}{$\begin{array}{c}>33 \mathrm{yr} \text { old, G2P1, GA } \\
33 \mathrm{w}\end{array}$} & \multirow[t]{3}{*}{ Choi et al. (2000) } & $\begin{array}{l}\text { (1) New onset hirsutism } \\
\text { @ GA } 28 \mathrm{w} \\
\text { (2) USS-enlarged right } \\
\text { ovary } \\
\text { (3) elevated maternal } \\
\text { testosterone }\end{array}$ & $\begin{array}{c}\text { Spontaneous preterm labor } \\
\qquad \text { GA 29w }\end{array}$ & Female infant & $\begin{array}{c}\text { USA } \\
\text { (Hispanic) }\end{array}$ \\
\hline & & & $\begin{array}{l}\text { (1) Abnormal results of } \\
\text { triple screen test }\end{array}$ & $\begin{array}{l}\text { Surgery: left oophorectomy } \\
\text { (luteoma of pregnancy) and } \\
\text { right anterior mass identified } \\
\text { as left lobe of liver }\end{array}$ & & $\begin{array}{c}\text { USA } \\
\text { (white) }\end{array}$ \\
\hline & $\begin{array}{c}>30 \mathrm{yr} \text { old, G2P1, GA } \\
21 \mathrm{w}\end{array}$ & & $\begin{array}{l}\text { (2) USS-left adnexa mass } \\
\text { with right anterior } \\
\text { abnormal mass }\end{array}$ & Pregnancy left in situ & $\begin{array}{c}\text { Pregnancy } \\
\text { ongoing at } \\
\text { time case } \\
\text { publication }\end{array}$ & \\
\hline 17 & $\begin{array}{c}>33 \text { yr old, G1P0, GA } \\
19 \mathrm{w}\end{array}$ & $\begin{array}{l}\text { Khurana et al. } \\
\text { (2017) }\end{array}$ & $\begin{array}{l}\text { (1) Incidental USS } \\
\text { finding and follow up } \\
\text { MRI-unilateral ovarian } \\
\text { mass }\end{array}$ & $\begin{array}{c}\text { Surgery: explorative } \\
\text { laparotomy and left } \\
\text { oophorectomy (dx after as } \\
\text { luteoma of pregnancy) and } \\
\text { pregnancy left in situ }\end{array}$ & $\begin{array}{c}\text { A term baby at } \\
40 \text { weeks }\end{array}$ & USA \\
\hline 18 & $\begin{array}{l}>28 \text { yr old, G4P3, } \\
\text { amenorrhoea } 2 / 12\end{array}$ & $\begin{array}{l}\text { Rathore et al. } \\
\qquad(2017)\end{array}$ & $\begin{array}{l}\text { (1) Presented with signs } \\
\text { of ectopic pregnancy and } \\
\text { USS showed tuboovarian } \\
\text { mass }\end{array}$ & $\begin{array}{c}\text { Surgery: emergency } \\
\text { laparotomy and salpingo- } \\
\text { oophorectomy }\end{array}$ & Not applicable & India \\
\hline 19 & $\begin{array}{c}>40 \mathrm{yr} \text { old, G2P1, GA } \\
16 \mathrm{w}\end{array}$ & $\begin{array}{l}\text { Wadzinski et al. } \\
\text { (2014) }\end{array}$ & $\begin{array}{l}\text { (1) Increasing acne, facial } \\
\text { hair, and deepening of } \\
\text { voice } \\
\text { (2) Lab: elevated } \\
\text { testosterone } \\
\text { (3) USS: negative for } \\
\text { mass }\end{array}$ & $\begin{array}{l}\text { Surgery: C-section of twins } \\
\text { @ } 33 \text { w of GA due } \\
\text { to preeclampsia }\end{array}$ & $\begin{array}{l}\text { Twins, female } \\
\text { infants with } \\
\text { ambiguous } \\
\text { genitalia }\end{array}$ & $\begin{array}{c}\text { USA } \\
\text { (Caucasian) }\end{array}$ \\
\hline
\end{tabular}


TABle 1: Continued.

\begin{tabular}{|c|c|c|c|c|c|c|}
\hline $\begin{array}{l}\text { Case } \\
\#\end{array}$ & Patient & Author (year) & $\begin{array}{l}\text { Presenting symptom } \\
\& \text { investigations }\end{array}$ & Management offered & $\begin{array}{c}\text { Outcome } \\
\text { (fetal genitalia } \\
\text { effects) }\end{array}$ & $\begin{array}{l}\text { Country } \\
\text { (race) }\end{array}$ \\
\hline \multirow[t]{2}{*}{20} & \multirow[t]{2}{*}{$>33$ yr old, GA $17 \mathrm{w}$} & \multirow{2}{*}{$\begin{array}{l}\text { Verma et al. } \\
\quad(2016)\end{array}$} & (1) Asymptomatic & $\begin{array}{l}\text { Surgery: C-section and } \\
\text { unilateral oophorectomy } \\
\text { suspecting malignancy }\end{array}$ & \multirow[t]{2}{*}{ Female infant } & \multirow[t]{2}{*}{ India } \\
\hline & & & $\begin{array}{l}\text { (2) Incidentally identified } \\
\text { intraoperatively }\end{array}$ & & & \\
\hline
\end{tabular}

(1) Hair on face and abdomen, deepened voice
UK

(2) USS done $4 \mathrm{w}$ postpartum: enlarged ovaries

$21>25$ yr old, GA $37 \mathrm{w}$ Holt et al. (2005)

(3) 3 yr later on another pregnancy above symptoms recurred

$2^{\text {nd }}$ pregnancy: SVD @ 39w Male infant

(4) Repeat USS done $5 \mathrm{w}$ postpartum: normal sized ovaries

(1) @ 5 k GA abdominal pain and USS: normal gestational sac with enlarged right ovary

Surgery: laparotomy and Csection (due to fetal distress and raptured membranes)

Female infant with ambiguous Italy

(2) @ $20 \mathrm{w}:$ abdominal pain and USS: both

\section{$22>34 \mathrm{yr}$ old,} primigravida

Mazza et al. (2002) ovaries enlarged

(3) Last 3/12 of pregnancy: increased abdominal pain, lower extremity hair, deepening of voice, and clitoromegaly
$>29$ yr old, primigravida, $\mathrm{PIH}$
Dhar et al. (2019) Incidental operative
finding

Surgery: emergency C-section due to fetal distress and "fibroid like mass" was Not mentioned India excised

(1) Febrile for $2 \mathrm{w}$, abdominal distension $4 / 7$

Spontaneous miscarriage @ $17 \mathrm{w}$ due to cervical Male fetus India
incompetence.

(2) labs: testosterone was elevated 30 times. Other labs were normal or inconclusive

$>26$ yr old,

24 primigravida@8w

GA
(3) USS; bilateral ovarian masses, moderate ascites and pleural effusion

(4) Laparoscopy: enlarged ovaries

(5) Frozen section suggested luteoma

(6) Maternal hirsutism was conspicuous@16w

Abbreviations: yr: year; GA: gestation age; MRI: magnetic resonance imaging; USS: ultrasound; TUSS: transvaginal ultrasound; @: at; nil: means no feminizing features; Labs: laboratory findings; +: means with or and; PCOD: polycystic ovarian disease; IVF: in vitro fertilization; PIH: pregnancy induced hypertension. 
development, the placenta is somewhat protective against the masculinization of the female fetus by converting the excess androgens to estrogens [5]. Emerging evidence has implicated androgenemia, specifically elevated testosterone, as a causative for preeclampsia [28]. Furthermore, preeclamptic women have been reported to have high placental expressions of the androgen receptor (AR) gene and elevated levels of testosterone two- to threefolds compared nonpreeclamptic women [28]. A finding that was consistent with our case report, she had hirsutism and preeclampsia. The only limitation in our case report was we failed to analyze the level of testosterone.

The diagnosis of pregnancy luteoma requires a high index of suspicion; once you suspect it based on the above-mentioned signs, perform detailed obstetric ultrasonography to visualize the size of the ovary. In addition, supportive investigations like hormonal assays should also be done to rule out other ovarian neoplasms [29].The management of pregnancy luteoma is case dependent (Table 1), for instance, cases presenting with severe virilization symptoms [2], pressure symptoms, or torsion will require prompt surgery while the asymptomatic cases require conservative managements with regular follow-up [30] as they usually regress spontaneously following delivery. Despite lacking hormonal assays in this case, spontaneous regression of hyperandrogenemia is the natural course of pregnancy luteoma [5]. Based on the presently described case, surgery for pregnancy luteoma was not indicated since there were no distressed symptoms. Instead, proper imaging techniques, preferably ultrasound or MRI and exploration of hormonal profile would suffice for diagnosis and management.

\section{Conclusions}

Based on the presently described case, pregnancy luteoma is a benign neoplasm and imprudent surgical intervention should be reserved. A high index of suspicion is paramount to diagnose pregnancy luteoma, and once suspected, one should perform a detailed obstetric ultrasound visualizing the size of the ovary and hormonal assay for testosterone and its derivatives.

\section{Conflicts of Interest}

The authors have no conflicts of interest to declare.

\section{Acknowledgments}

The authors are sincerely grateful to the patient for allowing us to share this informative report. We are also grateful for the excellent technical assistance from Dr. Nimish Chayya, Consultant Pathologist at TMJ Hospital, and the Departments of $\mathrm{O} \& \mathrm{G}$ at Lugalo Military Hospital and Muhimbili University of Health and Allied Sciences. We are forever indebted for the continued support and encouragement from the $\mathrm{O} \& \mathrm{G}$ postgraduate class of 2018 .

\section{References}

[1] E. A. M. Kuijper, J. C. F. Ket, M. R. Caanen, and C. B. Lambalk, "Reproductive hormone concentrations in pregnancy and neonates: a systematic review," Reproductive Biomedicine Online, vol. 27, no. 1, pp. 33-63, 2013.

[2] V. Rapisarda, F. Pedalino, V. C. Santonocito, G. Cavalli, and G. Zarbo, "Luteoma of pregnancy presenting with severe maternal virilisation: a case report," Case Reports in Obstetrics and Gynecology, vol. 2016, 4 pages, 2016.

[3] W. H. Sternberg and D. L. Barclay, "Luteoma of pregnancy," American Journal of Obstetrics and Gynecology, vol. 95, no. 2, pp. 165-184, 1966.

[4] K. Masarie, V. Katz, and K. Balderston, "Pregnancy luteomas," Obstetrical \& Gynecological Survey, vol. 65, no. 9, pp. 575-582, 2010.

[5] Y. C. Wang, H. Y. Su, J. Y. Liu, F. W. Chang, and C. H. Chen, "Maternal and female fetal virilization caused by pregnancy luteomas," Fertility and Sterility, vol. 84, no. 2, pp. 509.e15509.e17, 2005.

[6] D. S. Ranjan, R. V. Kumar, B. Rao, and E. S. Rao, "Maternal luteoma of pregnancy: a rare case report," Journal of Dr. NTR University of Health Sciences, vol. 3, no. 4, p. 267, 2014.

[7] J. F. K. Tannus, B. S. Hertzberg, C. M. Haystead, and E. K. Paulson, "Unilateral luteoma of pregnancy mimicking a malignant ovarian mass on magnetic resonance and ultrasound," Journal of Magnetic Resonance Imaging, vol. 29, no. 3, pp. 713-717, 2009.

[8] A. Nanda, U. A. Gokhale, and G. R. Pillai, "Bilateral pregnancy luteoma: a case report," Oman Medical Journal, vol. 29, no. 5, pp. 371-372, 2014.

[9] R. K. Brar, J. N. Bharti, J. S. Nigam, S. Sehgal, H. P. Singh, and P. Blair, "Pregnancy luteoma in ectopic pregnancy: a case report," Journal Reproduction \& Infertility, vol. 18, no. 3, pp. 333-335, 2017.

[10] C. Roth, B. Hinney, M. Peter, D. Steinberger, and M. Lakomek, "Features of Antley-Bixler syndrome in an infant born to a mother with pregnancy luteoma," European Journal of Pediatrics, vol. 159, no. 3, pp. 189-192, 2000.

[11] R. F. Spitzer, D. Wherrett, D. Chitayat et al., "Maternal luteoma of pregnancy presenting with virilization of the female infant," Journal of Obstetrics and Gynaecology Canada, vol. 29, no. 10, pp. 835-840, 2007.

[12] H. Ugaki, T. Enomoto, Y. Tokugawa, and T. Kimura, "Luteoma-induced fetal virilization," The Journal of Obstetrics and Gynaecology Research, vol. 35, no. 5, pp. 991-993, 2009.

[13] M. L. Tan, S. L. Lam, and S. Nadarajah, "Pregnancy luteoma presenting as ovarian torsion with rupture and intraabdominal bleeding," Singapore Medical Journal, vol. 49, no. 3, 2008 .

[14] J. R. Choi, D. Levine, and H. Finberg, "Luteoma of pregnancy: sonographic findings in two cases," Journal of Ultrasound in Medicine, vol. 19, no. 12, pp. 877-881, 2000.

[15] A. Banerjee, F. Tahmasebi, E. Myola, and W. Yoong, "Luteoma of pregnancy," Journal of Obstetrics and Gynaecology, vol. 26, no. 6, pp. 572-574, 2009.

[16] Bilateral pregnancy luteoma - a case report, 2019, https://www .emedinexus.com/post/10111/bilateral-pregnancy-luteomaa-case-report.

[17] S. K. Dahl, M. A. Thomas, D. B. Williams, and J. C. Robins, "Maternal virilization due to luteoma associated with delayed 
lactation," Fertility and Sterility, vol. 90, no. 5, pp. 2006.e172006.e19, 2008.

[18] V. Verma, S. Paul, K. Chahal, and J. Singh, "Pregnancy luteoma: a rare case report," International Journal of Applied and Basic Medical Research, vol. 6, no. 4, pp. 282-283, 2016.

[19] S. Dasari, P. Rangaram, S. R. Gundabattula, and E. Joseph, "Bilateral luteomas of pregnancy," Journal of Obstetrics and Gynaecology, vol. 33, no. 5, p. 521, 2013.

[20] A. Khurana and M. O’Boyle, "Luteoma of pregnancy," Ultrasound Quarterly, vol. 33, no. 1, pp. 90-92, 2017.

[21] P. Masquerading, E. Pregnancy, and L. Learnt, "Luteoma of pregnancy masquerading as ectopic pregnancy : lessons learnt," Saudi Journal of Medicine and Medical Sciences, vol. 5, no. 3, pp. 281-283, 2017.

[22] T. L. Wadzinski, Y. Altowaireb, R. Gupta, R. Conroy, and K. Shoukri, "Luteoma of pregnancy associated with nearly complete virilization of genetically female twins," Endocrine Practice, vol. 20, no. 2, pp. 18-23, 2014.

[23] H. B. Holt, "Recurrent severe hyperandrogenism during pregnancy: a case report," Journal of Clinical Pathology, vol. 58, no. 4, pp. 439-442, 2005.

[24] V. Mazza, I. di Monte, P. L. Ceccarelli et al., "Prenatal diagnosis of female pseudohermaphroditism associated with bilateral luteoma of pregnancy: case report," Human Reproduction, vol. 17, no. 3, pp. 821-824, 2002.

[25] R. Dhar, P. Gaikwad, and S. Sahu, "Luteoma of pregnancy: a case report," Int. J. Res. Rev, vol. 6, pp. 21-23, 2019.

[26] M. Deknuydt, A. Dumont, A. Bruyneel, D. Dewailly, and S. Catteau-Jonard, "Recurrent maternal virilization during pregnancy in patients with PCOS: two clinical cases," Reproductive Biology and Endocrinology, vol. 16, no. 1, p. 107, 2018.

[27] M. N. A. Salloum, A. M. Hakoun, I. AbouAl-Shaar, K. J. Zaza, and H. Abou-Al-Shaar, "Adnexal masses in pregnancy: an updated review,” Avicenna Journal of Medicine, vol. 7, no. 4, pp. 153-157, 2017.

[28] S. Kumar, G. H. Gordon, D. H. Abbott, and J. S. Mishra, "Androgens in maternal vascular and placental function: implications for preeclampsia pathogenesis," Reproduction, vol. 156, no. 5, pp. R155-R167, 2018.

[29] E.-S. Song, J.-P. Lee, J.-H. Han et al., "Dysgerminoma of the ovary with precocious puberty: a case report," Gynecological Endocrinology, vol. 23, no. 1, pp. 34-37, 2009.

[30] S. A. Duru Coteli, G. Orgul, and M. C. Salman, "Pregnancy luteoma: a rare presentation and expectant management," Case Reports in Perinatal Medicine, vol. 7, no. 2, 2018. 